\title{
La Finlande et la Suède, des pays encore non-alliés ? Cultures et politiques de sécurité à l'épreuve des tensions régionales dans l'espace baltique
}

\author{
Sophie ENOS-ATTALI \\ Maître de conférences \\ Faculté de Sciences Sociales et Économiques \\ Institut catholique de Paris \\ s.enos-attali@icp.fr
}

\begin{abstract}
Résumé
«Petits pays » d'Europe du Nord, la Finlande et la Suède ont chacune fait le choix de la neutralité pour assurer leur sécurité dans un contexte où elles se sentaient fragilisées par leur voisinage avec la Russie. Après l'effondrement de l'Union soviétique au sortir de la Guerre froide, elles ont mené une politique originale, associant la participation pleine et entière à la politique étrangère et de sécurité commune de l’Union européenne et la non-participation à des alliances militaires. Toutefois, bouleversant en profondeur l'équilibre en Europe du Nord, la crise ukrainienne a conduit la Finlande et la Suède à prendre leurs distances avec l'objectif de neutralité dans un conflit armé, qui avait été au cœur de leur politique sécuritaire depuis les années 1950. Par-delà ce qui apparait comme une rupture avec leur tradition sécuritaire, la Finlande et la Suède demeurent en réalité fidèles à leur culture de sécurité : comme par le passé, c'est l'image qu'elles ont chacune d'elles-mêmes, une image demeurée inchangée, qui continue d'encadrer leurs choix sécuritaires.
\end{abstract}

Mots-clés : Finlande, Suède, neutralité, non-alliance, culture de sécurité

\begin{abstract}
As "small countries" of Northern Europe, Finland and Sweden each chose neutrality to ensure their security in a context where they felt weakened by their proximity to Russia. After the collapse of the Soviet Union and at the end of the Cold War, they pursued an original policy, combining full participation in the European Union's Common Foreign and Security Policy and non-participation in military alliances. However, the Ukrainian crisis disrupted the balance in Northern Europe, leading Finland and Sweden to distance themselves from neutrality in armed conflicts, although this objective had been at the heart of their security policy since the 1950s. Beyond what appears to be a break with their security tradition, both countries remain in reality faithful to their security culture: as in the past, their security choices are determined by their self-image, and this image has remained unchanged.
\end{abstract}

Keywords: Finland, Sweden, neutrality, non-alliance, security culture. 
1. La neutralité, une composante des cultures de sécurité de la Finlande et de la Suède ? 159

1.1 Les neutralités finlandaise et suédoise : des origines différentes

1.2 Durant la Guerre froide, deux pratiques distinctes de la neutralité

1.3 La fidélité de la Finlande et de la Suède au principe de non-alliance après la Guerre froide, signe d'un ancrage de la neutralité dans les deux pays?

2. L'héritage de la neutralité à l'épreuve de la crise ukrainienne

2.1 Un environnement menaçant

2.2 Des politiques de sécurité renouvelées

2.2.1 Des postures de sécurité ambiguës

2.2.2 Des capacités de défense renforcées

2.2.3 Des pays engagés dans la coopération internationale

2.3 Par-delà les tensions régionales, des politiques de sécurité inchangées?

2.3.1 Le pragmatisme au cœur de la politique de sécurité finlandaise

2.3.2 La politique de sécurité suédoise à un carrefour

Conclusion 


\section{La Finlande et la Suède, des pays encore non-alliés ? Cultures et politiques de sécurité à l'épreuve des tensions régionales dans l'espace baltique}

\section{Introduction}

Au regard de leur superficie (respectivement $450295 \mathrm{~km}^{2}$ et $338424 \mathrm{~km}^{2}$ ) et de leur population (près de 10 millions de Suédois et 5,5 millions de Finlandais), la Suède et la Finlande font figure, en Europe, de pays de taille modeste. Cette caractéristique, ajoutée à leur proximité avec de grandes puissances, en particulier la Russie, et à leur passé tumultueux, les a conduites toutes deux, dans des contextes historiques néanmoins différents, à fonder leur politique de sécurité sur le principe de neutralité. Désignant le fait pour un État de demeurer en dehors d'un conflit, la neutralité entraîne, pour celui qui s'en réclame de manière durable, un certain nombre de devoirs parmi lesquels l'interdiction de conclure un accord susceptible de l'entraîner dans une guerre, ainsi que l'impératif d'assurer seul sa défense. Si la Finlande et la Suède ne se sont pas toujours pliées scrupuleusement à ces obligations, n'hésitant pas à transiger, en particulier durant la Guerre froide, avec leur devoir de défense autonome, elles n'en ont pas moins érigé la non-participation à des alliances militaires en vue de la neutralité en temps de guerre en ligne directrice de leur politique de sécurité. Or, comme l'explique Laurent Goetschel, la neutralité, parce qu'elle est associée, par définition, à la sécurité nationale et qu'elle est perçue comme porteuse de valeurs positives comme la paix, tend à être idéalisée par les sociétés des pays de tradition neutre, se trouvant ainsi intériorisée comme une norme et donc naturellement intégrée à leur culture de sécurité (Goetschel 1999, 131-133).

Définie par Mary Kaldor comme "a specific pattern of behaviour, or constellation of socially meaningful practices, that expresses or is the expression of norms and standards embodied in a particular interpretation of security" (Kaldor 2018, 1-2), la culture de sécurité suppose que sont enracinés, dans la culture politique d'un pays, des narratifs, des croyances, des règles, des outils, des façons de percevoir le monde et de concevoir les choses, des manières de faire qui survivent aux circonstances qui les ont fait émerger et encadrent l'action politique en matière de sécurité. Dans cette perspective, la politique de sécurité d'un pays ne découlerait pas seulement « d'une évaluation purement rationnelle de contraintes internationales et capacités nationales objectivement données » (Goetschel 1998, 30), mais serait en partie déterminée par des facteurs culturels (Katzenstein 1996, 
22-26). De sorte qu'en l'absence d'événement majeur modifiant en profondeur l'équilibre stratégique, toute politique nationale de sécurité, se trouvant contrainte par le patrimoine cognitif que représente la culture de sécurité, ne changerait qu’à la marge (Longhurst 2004, 17). En d'autres termes, l'ancrage de certaines normes et pratiques dans la culture de sécurité d'un État impliquerait une relative stabilité de la politique de sécurité de cet État. Le fait que la Finlande et la Suède soient demeurées fidèles au principe de non-participation à des alliances militaires en vue de la neutralité par-delà la dislocation de l'Union soviétique et l'adhésion des pays baltes à l'Alliance atlantique ${ }^{1}$ tend à asseoir l'hypothèse d'une inscription de l'objectif de neutralité et de son corollaire, la non-alliance, dans leur culture de sécurité respective.

Cependant, les choix récents d'Helsinki et de Stockholm en matière de sécurité invitent à nuancer cette lecture. De fait, dans le contexte de la crise ukrainienne qui voit la Russie augmenter ses capacités militaires en Europe du Nord et multiplier les provocations à l'encontre des pays riverains de la Baltique, la Finlande et la Suède, tout en demeurant formellement en dehors de toute alliance militaire, arriment désormais ouvertement leur sécurité à la coopération internationale, prenant ainsi très nettement leurs distances par rapport à leur tradition de neutralité, à laquelle elles ne se réfèrent d'ailleurs plus. Faut-il voir dans cette évolution un tournant dans les politiques de sécurité des deux pays ou la fidélité au principe de non-alliance reflète-t-elle l'ancrage de la neutralité dans les cultures politiques finlandaise et suédoise ? C'est à cette question que nous tâcherons de répondre, en replaçant les politiques de sécurité de la Finlande et de la Suède dans une perspective historique. Dans un premier temps, nous nous intéresserons aux origines des neutralités finlandaise et suédoise et étudierons les politiques de sécurité menées par Helsinki et Stockholm entre le début du conflit bipolaire et la fin des années 2000. Puis, dans une seconde partie, nous analyserons les choix faits par la Finlande et la Suède en matière de sécurité dans le cadre de l'annexion de la Crimée par la Russie. Cette étude comparative de l'évolution des discours et des pratiques de sécurité de la Finlande et de la Suède depuis la Guerre froide nous permettra ainsi de mettre en évidence les ressorts des politiques de sécurité des deux pays.

1 En 2004, l'Estonie, la Lettonie et la Lituanie ont adhéré à l'Organisation du traité de l'Atlantique nord (OTAN). 
1. La neutralité, une composante des cultures de sécurité de la Finlande et de la Suède?

Fondées sur l'héritage d'histoires mouvementées et sur une position géographique source de vulnérabilité, les politiques de sécurité de la Finlande et de la Suède reposent historiquement sur une tradition de neutralité qui, pour des raisons distinctes et de manière différente, s'est progressivement ancrée dans l'imaginaire collectif des deux pays, se trouvant ainsi plébiscitée jusqu'à la fin de la Guerre froide.

\subsection{Les neutralités finlandaise et suédoise : des origines différentes}

La Suède et la Finlande ont adopté la neutralité dans des contextes historiques sensiblement différents, de sorte qu'elle ne s'inscrit pas de la même manière dans la culture de sécurité des deux pays.

Si la Suède a pu chercher occasionnellement (en 1691 et 1780) à adopter une ligne neutre, elle s'est néanmoins trouvée, tout au long des XVII ${ }^{\mathrm{e}}$ et XVIII ${ }^{\mathrm{e}}$ siècles, impliquée dans plusieurs conflits, rejoignant même, en 1803, la coalition antinapoléonienne. C'est finalement devant l'émergence d'un État allemand unifié et face à l'affirmation de la Russie et du Royaume-Uni en Europe que le roi Charles XIV Jean, conscient des faiblesses structurelles du pays par rapport à ses puissants voisins, choisit, dès qu'il accède au trône, en 1818, d'asseoir la sécurité suédoise sur une politique de neutralité durable. Soucieux de garantir au pays le maintien de sa liberté et de son indépendance et de lui assurer la sécurité, il affirme ainsi la volonté systématique de rester en dehors de toute guerre. Pour autant, dans les faits, la politique de sécurité de la Suède est fluctuante : de la première guerre du Schleswig² (1848-1850) à la Seconde Guerre mondiale (1939-1945), Stockholm fait quelques entorses à la neutralité, n’hésitant pas, selon les circonstances, à s'engager aux côtés d'un pays ou d'un autre, par exemple en autorisant la flotte anglo-française à utiliser ses ports et ses bases pour conduire des attaques contre des positions russes durant la guerre de Crimée $^{3}$ (1853-1856) ou encore en

2 Cette guerre a opposé la Confédération germanique au Danemark, dont le roi, Frédéric VII, avait décidé d'incorporer à son royaume le duché du Schleswig, peuplé d'Allemands et de Danois. Au nom de la solidarité scandinave et d'une histoire longtemps commune, le roi de Suède s'est déclaré prêt à soutenir toute puissance qui interviendrait pour maintenir les droits du Danemark sur le Schleswig, tandis que des volontaires suédois partaient combattre sous le drapeau danois. Après la signature de la paix à Berlin, le 2 juillet 1850 , cette guerre s'est soldée par la conclusion, à Londres, en 1851 et 1852, de protocoles qui ont rendu l'administration du Schleswig, mais aussi des duchés de Holstein et de Saxe-Lauenbourg au Danemark, tout en garantissant leur autonomie.

3 Provoquée par la prétention de Moscou à assurer en exclusivité la protection des lieux saints de Jérusalem, la guerre de Crimée oppose l'Empire russe à une coalition formée par l'Empire ottoman, le Royaume-Uni, l'Empire de Napoléon III et le royaume de Sardaigne. 
adoptant plusieurs mesures favorables à l'Allemagne nazie dès $1940^{4}$, avant de prendre part au blocus contre cette dernière en 1943. Malgré cela, considérée comme un rempart efficace contre toute menace car elle a permis au pays de demeurer en paix par de-delà les turbulences de l'histoire, la neutralité se trouve érigée en pilier de la politique de sécurité suédoise. Une tendance que conforte la bipolarité : craignant que Moscou ne s'en prenne à la Finlande si elle prenait ellemême trop de libertés avec la neutralité, la Suède affiche sa fidélité à sa tradition sécuritaire, faisant de la non-participation à une alliance militaire le principe fondamental de sa politique de sécurité. De sorte qu'au fil du temps, la neutralité en vient à constituer un élément à part entière de la culture politique du pays (Miles 1995, 17).

Côté finlandais, c'est la relation complexe avec la Russie qui est à l'origine de la neutralité. Devenue grand-duché autonome de l'Empire russe à la suite de la guerre russo-suédoise de 1808-1809 qui met fin à son rattachement à la Suède, la Finlande se trouve entraînée dans la guerre de Crimée, une expérience douloureuse qui nourrit l'idée selon laquelle la nation finlandaise doit s'affirmer vis-à-vis de Moscou, notamment en développant la capacité de se défendre seule, sans aide extérieure, pour ne plus être à la merci des puissances étrangères et, surtout, cesser d'être un gage entre la Russie et ses adversaires (Nevakivi 1989). Toutefois, le pays connait, dans les années 1890, une vague de russification, qui aboutit, en 1901, à la formulation, par l'historien et parlementaire Johan-Richard Danielson-Kalmari, d'une doctrine de sécurité dite de "soumission limitée », selon laquelle le pays ne serait jamais en danger tant qu'il maintiendrait de bonnes relations avec la Russie. C'est finalement en 1920 que la Finlande, qui a proclamé son indépendance le 6 décembre 1917, à la faveur des désordres occasionnés par la Première Guerre mondiale, envisage d'adopter une ligne de neutralité : Helsinki conclut ainsi en 1932 un traité de non-agression et de neutralité avec Moscou, puis signe en 1933 le Traité d'Oslo, qui établit une zone nordique de coopération dans la neutralité. Toutefois, elle rompt avec cette posture durant la Seconde Guerre mondiale en recourant à l'aide de l'Allemagne nazie dans la guerre de Continuation (1941-1944) qui l'oppose à l'Union soviétique ${ }^{5}$. Si elle parvient à

4 Bien qu'officiellement neutre, la Suède a maintenu des relations économiques soutenues avec l'Allemagne nazie qui a été, de 1940 à 1943, son principal partenaire économique, et a permis le transit, par son territoire, de soldats allemands.

5 Traumatisée par la guerre d'Hiver (30 novembre 1939-12 mars 1940), par laquelle l'Union soviétique est parvenue à s'emparer de territoires finlandais importants, la Finlande, dans l'espoir de reconquérir ses terres, a lutté aux côtés de l'Allemagne nazie quand cette dernière a envahi l'URSS en 1941. Cette guerre, dite " de Continuation ", s'est terminée en septembre 1944 par la signature d'un armistice en vertu duquel la Finlande rompt avec le III ${ }^{e}$ Reich et cède à l'Union soviétique non seulement les territoires déjà perdus en 1940, mais aussi la région de Petsamo. 
rester souveraine au sortir de la guerre, elle fait néanmoins l'objet de sanctions qui la placent dans une situation d'indépendance limitée, puisque le Traité de Paris (1947) fixe des limites aux effectifs de l'armée finlandaise et, surtout, interdit à Helsinki de conclure des traités d'alliance dirigés contre l'une des parties contractantes. À la suite de cela, convaincu que la Finlande ne peut échapper à la puissance soviétique, le président Juho Kusti Paasikivi conclut avec le Kremlin, le 6 avril 1948, un traité d'amitié, de coopération et d'assistance mutuelle qui, tout en mentionnant le désir d'Helsinki de rester en dehors des « intérêts antagonistes des grandes puissances ", prévoit que le pays empêche tout envahisseur étranger d'utiliser son territoire pour conduire une agression contre l'Union soviétique et, à cette fin, ne conclue aucune alliance dirigée contre Moscou et accepte l'aide militaire soviétique en cas de menace ${ }^{6}$. C'est dans ce contexte que la Finlande adopte, au milieu des années 1950, une politique de neutralité, en se tenant à l'écart de toute alliance militaire et en développant les capacités militaires de se défendre seule.

Les politiques de sécurité de la Suède et de la Finlande s'ancrent donc dans la neutralité, mais pour des raisons différentes : alors qu'à l'origine le choix de la neutralité par Stockholm traduit l'image que la Suède a d'elle-même, celle d'un petit pays qui a intérêt à favoriser l'émergence d'un équilibre des puissances et à composer avec ces dernières, c'est le sentiment de vulnérabilité et le souci de la Finlande de s'affirmer comme un État pleinement souverain face à la Russie puis à l'URSS qui conduit Helsinki à opter pour la neutralité.

Compte tenu de ces différences, la neutralité ne s’inscrit pas de la même manière dans la culture politique des deux pays. En Suède, la posture de neutralité, en raison de son adoption ancienne, mais aussi de l'histoire du pays, de rester en dehors de tout conflit, est considérée comme une tradition à laquelle il faut rester fidèle afin de demeurer en paix. En Finlande, la neutralité est davantage appréhendée comme un instrument au service du maintien de la souveraineté du pays. De sorte que si on observe, dans les deux nations, un attachement fort à la neutralité, les raisons en sont différentes : les Suédois considèrent la neutralité comme une composante de leur identité nationale là où les Finlandais s'y accrochent essentiellement par pragmatisme car ils y voient un outil d'indépendance et de sécurité nationales. Ces différences ne sont pas sans implication sur leur politique de sécurité pendant la Guerre froide.

6 Voir "The Agreement of Friendship, Cooperation, and Mutual Assistance between The Union of Soviet Socialist Republics and The Republic of Finland" 


\subsection{Durant la Guerre froide, deux pratiques distinctes de la neutralité}

Lorsque débute la Guerre froide, la Finlande et la Suède se trouvent dans une situation différente: alors que Stockholm est forte d'une tradition ancienne de neutralité, qui trouve son origine dans un souci d'équilibre stratégique, la Finlande est liée à l'URSS par un traité qui, tout en affirmant la nécessité pour le pays de demeurer en dehors d'une alliance militaire, prévoit des consultations sécuritaires avec Moscou. Si elles sont toutes deux tenues aux mêmes obligations en tant qu'États neutres, en particulier l'impératif d'assurer seules leur défense et l'interdiction de conclure un traité ou une alliance susceptible, à terme, d'entraîner leur participation à une guerre, dans les faits, elles donnent chacune une coloration singulière à leur politique de neutralité durant la Guerre froide.

En premier lieu, les deux pays n’interprètent pas exactement de la même manière les implications politiques de la neutralité. Se réclamant des valeurs occidentales, la Suède ne considère pas que la neutralité l'empêche de prendre parti politiquement dans la Guerre froide : c'est ainsi que, bénéficiaire du plan Marshall, elle participe en 1948 à l'établissement de l'Organisation européenne de coopération économique (OECE), puis, en 1960, à l'Association européenne de libre-échange (AELE), et est même candidate, en 1967, à l'adhésion à la Communauté économique européenne (CEE). De leur côté, les présidents finlandais Juho Paasikivi (1946-1956) et Urho Kekkonen (1956-1981), exploitant le souvenir cuisant de la guerre d'Hiver (Browning 2002, 48 ; Archer 2003, 61), définissent une ligne de politique étrangère et de sécurité qui met l'accent sur la nécessité de maintenir des relations de confiance avec l'Union soviétique et, dans cette perspective, de ne rien faire qui puisse froisser Moscou. Cette posture, désignée par le terme "finlandisation ${ }^{7}$, a ainsi conduit la Finlande à refuser l'aide Marshall, à ne pas prendre part à l'AELE et à considérer impossible une candidature à la CEE dans le contexte de la bipolarité.

Sur le plan militaire, la Finlande et la Suède diffèrent également. Si la Suède affirme poursuivre l'objectif d'une défense forte et indépendante, dans les faits, elle considère, dès le début de la Guerre froide, qu'elle n'est pas en mesure, en tant que petit pays insulaire, de combattre seule une grande puissance. Dans cette perspective, contrevenant au principe de neutralité, elle coordonne en secret, dès 1948, la planification de sa défense avec les États-Unis, le Royaume-Uni, le

7 Utilisé en 1953 par le ministre des Affaires étrangères autrichien Karl Gruber en référence aux relations finnosoviétiques découlant du Traité d'amitié de 1948, le terme " finlandisation » désigne, au-delà de la soumission de la Finlande à l'Union soviétique durant la Guerre froide : « une ingérence sournoise dans le fonctionnement interne d'un pays qui arrive à le bloquer s'il ne va pas comme on le désire » (Thibaut 1990, 157). 
Danemark et la Norvège, tous quatre membres-fondateurs de l'Organisation du traité de l'Atlantique nord (OTAN) : reposant sur une coopération technologique et militaire, ainsi qu'en matière de renseignement, ce "partenariat » doit permettre au pays de bénéficier d'une assistance occidentale en cas de besoin (Molin 2000, 264-266 ; Winnerstig 2001, 76-77). Puis, à la fin des années 1950, estimant ne pouvoir faire l'objet d'une attaque de grande envergure en raison de lintérêt stratégique limité de son territoire, elle décide de passer d'une stratégie de défense totale à une stratégie de défense marginale ${ }^{8}$, ce qui, en réalité, porte un coup sérieux à sa capacité à assurer seule sa défense. La Finlande, elle, s'attache davantage que sa voisine scandinave à respecter l'obligation de défense efficace découlant de la neutralité : elle alloue ainsi à sa politique de dissuasion des moyens croissants qui lui permettent d'acquérir assez rapidement un bon niveau de défense. En revanche, si les dirigeants finlandais veillent à un certain équilibre entre l'Est et l'Ouest dans l'acquisition d'armements, l'establishment militaire noue, à partir de 1962, des contacts avec les États-Unis : ces derniers transfèrent ainsi savoir-faire et équipement de reconnaissance à la Finlande, tandis que des plans prévoient un soutien américain à Helsinki en cas de crise (Heikka 2005a ; Ganser 2005).

Durant la Guerre froide, les deux pays entendent s'affirmer comme acteurs de paix, mais ils ne le font pas dans la même mesure : alors que la Suède, en particulier sous l'égide de son premier ministre Olof Palme (1969-1976 et 1982-1986), se fait le chantre d'une politique de neutralité active basée sur la promotion de la sécurité collective et de la stabilité régionale, sur l'aide régionale au développement et sur l'action en faveur du contrôle des armements, la Finlande, se prévalant de son positionnement entre l'Est et l'Ouest, cherche en premier lieu à contribuer à la « détente », qui trouve son apogée avec la tenue, à Helsinki, en 1975, de la Conférence sur la sécurité et la coopération en Europe.

La pratique de la neutralité par la Finlande et la Suède durant la Guerre froide est donc révélatrice d'un rapport différent des deux pays à la neutralité : attachée davantage à l'esprit qu’à la lettre de la neutralité, Stockholm, pragmatique, privilégie sa sécurité sur le respect de la posture neutre tout en veillant à offrir l'image d'un pays activement engagé dans la paix, tandis que la Finlande est surtout préoccupée par sa proximité avec l'URSS, qu'elle veille à ne pas froisser. Toutefois, à la fin de la Guerre froide, on observe une certaine convergence dans le rapport des deux pays à la neutralité.

8 Alors que le concept de « défense totale » prévoit que « toutes les composantes de la société, aussi bien civiles que militaires, participent à l'effort de défense », en cas d'état d'alerte maximal, la " défense marginale » repose sur des capacités très réduites (Sydow 2018). 


\subsection{La fidélité de la Finlande et de la Suède au principe de non-alliance après la Guerre froide, signe d'un ancrage de la neutralité dans les deux pays ?}

Avec l'effondrement de l'Union soviétique, les deux pays nordiques, considérant ne plus avoir à craindre leur voisine russe, font sensiblement évoluer leur politique de sécurité. Cependant, alors même que la peur d'une attaque de la part de Moscou avait été en partie à l'origine de leur choix de la neutralité, aucun des deux pays ne renonce complètement à cette tradition une fois cette menace atténuée.

La Finlande, dès la seconde moitié des années 1990, et la Suède, au début des années 2000, abandonnent la référence à la neutralité comme fin en soi : considérant que la politique de neutralité suivie durant la Guerre froide n'est plus une ligne d'action encore viable, la Finlande évoque même la possibilité de recevoir une assistance militaire étrangère (Reports by the Council of State to the Parliament 1995 ; 1997), tandis que la Suède, tout en restant fidèle au principe de non-alliance, place la coopération internationale au cœur de sa politique de sécurité . Conformément à ces nouvelles postures, les deux pays intègrent l'Union européenne (UE) sans aucune réserve concernant la Politique étrangère et de sécurité commune (PESC) et prennent part dès 1997 au Partenariat pour la paix (PPP) établi par l'OTAN. Ils participent dès lors activement à plusieurs missions sous l'égide de l'UE comme de l'OTAN. Dans cette logique, la Finlande et la Suède veillent à l'interopérabilité de leur appareil militaire avec ceux des États membres de l'UE - qui sont pour la plupart également membres de l'OTAN - de manière à pouvoir prendre part à des exercices ainsi qu'à des opérations multinationaux dans le cadre de ces organisations. La Suède abrite même des centres d'entraînement militaires labellisés par le PPP.

Les deux pays œuvrent également au développement d'une coopération nordique en matière de défense. Est ainsi institutionnalisée en 2009 la Coopération nordique de défense (NORDFECO) avec la Norvège, le Danemark et l'Islande. De plus, à l'automne 2013, Helsinki et Stockholm annoncent leur intention de renforcer leur coopération de défense afin d’intensifier la sécurité dans la région et d'optimiser le rapport coût/efficacité. Concrètement, cela repose sur un programme d'exercices conjoints, sur la capacité des deux armées à accéder au territoire et à utiliser mutuellement leurs bases, sur la mise en place d'une unité mixte finno-suédoise pour participer à des exercices internationaux (Combined

9 Voir les déclarations de politique gouvernementale lors du débat parlementaire sur les affaires étrangères de 2002, 2003 et 2004. 
Finnish-Swedish Brigade Framework), sur le développement d'une force maritime finno-suédoise (Swedish Finnish Naval Task Force), ainsi que sur le déploiement d'un réseau de communication sécurisé pour faciliter l'échange d’informations classées (Szymanski 2020).

Parallèlement à cela, les deux pays réforment leur appareil de défense en vue d'une plus grande interopérabilité. La Suède, considérant dès la fin des années 1990 son environnement immédiat comme sûr et moins menaçant que durant la Guerre froide, fixe comme priorité à ses forces armées non plus la capacité de défense du territoire national, mais celle de participer à des missions de gestion de crise. Dans cette perspective, elle démantèle des installations de défense, ferme des bases militaires, supprime en 2010 la conscription obligatoire et réduit à 50000 soldats les effectifs de l'armée, pour concentrer ses efforts sur la modernisation de ses forces afin de favoriser leur participation aux opérations internationales de maintien de la paix. Entre 1995 et 2009, la part de son produit intérieur brut (PIB) consacrée à la défense passe de 2,2 \% à 1,2 \% (Nünlist 2013). Demeurant prudente en raison de sa frontière partagée avec la Russie, la Finlande, elle, réduit certes la part de son budget consacrée à la défense, mais maintient le service militaire et continue d'accorder de l'importance à la défense territoriale. Ainsi, en 2001, si la Suède est au $8^{\mathrm{e}}$ rang des pays de l'UE en termes de dépenses de défense, elle dispose en moyenne seulement de 3,8 soldats pour 1000 habitants, contre 6,2 pour la Finlande, laquelle occupe le $12^{\mathrm{e}}$ rang au sein de l'UE pour ce qui est des dépenses de défense.

Dans le même temps, par-delà ces évolutions, Helsinki et Stockholm n'en demeurent pas moins fidèles à ce qui constitue le cœur de la posture de neutralité, le principe de non-appartenance à des alliances militaires, dans lequel elles voient toujours un moyen d'assurer leur sécurité. Ainsi, si les deux pays ne mènent plus officiellement, ni en pratique, une politique de neutralité, notamment avec leur participation active à la PESC et à des opérations de paix sous l'égide de l'OTAN, le principe de non-alliance demeure un élément important de leur politique de sécurité.

Cette combinaison inédite entre non-alliance et participation active à la coopération internationale en matière de sécurité peut s'expliquer de plusieurs manières. D’une part, ne se sentant plus directement menacés, les deux pays choisissent de rester en dehors de l'OTAN afin de ne pas affecter la stabilité de l'Europe du Nord, mais aussi parce qu'ils n'estiment pas qu'une adhésion à l'alliance militaire renforcerait nécessairement leur sécurité dans un contexte perçu comme plus sûr. D'autre part, cette position d'entre-deux, ni alliés, ni isolés d'un point de 
vue sécuritaire, fait l'objet d'un consensus politique et rencontre les aspirations de la population. Ainsi, en Finlande, si quelques personnalités politiques se sont prononcées en faveur de l'abandon du principe de non-participation aux alliances militaires, en revanche, plus de la moitié des Finlandais souhaite que leur pays demeure non-allié (Clerc 2005, 44) et plus de $60 \%$ sont contre une adhésion à l'OTAN, comme le souligne Andrew Rettman dans EuObserver le 26 avril 2007. En Suède, si la question de l'adhésion à l'Europe communautaire a divisé la classe politique comme la population ${ }^{10}$, le non-alignement est en revanche plébiscité par une assez large majorité de Suédois ${ }^{11}$.

Quelque vingt ans après la Guerre froide, il semble donc bien exister, en Finlande comme en Suède, un héritage de la neutralité. Toutefois, celui-ci ne s’inscrit pas de la même manière dans les cultures de sécurité des deux pays. Si la Finlande a bien intégré certaines des pratiques liées à la neutralité, en particulier le principe de non-alliance et l'impératif d'assumer seule la responsabilité de sa défense (Archer 2003, 61), sa politique de sécurité semble avant tout guidée par la conscience aiguë d'une grande vulnérabilité face à la Russie, potentiellement instable (Heikka 2005b). Comme le résume Kyösti Karvonen, rédacteur en chef du journal Kalevala, les Finlandais sont intimement convaincus que leur pays « défend au mieux ses intérêts en gardant un profil bas tout en évitant soigneusement de s'immiscer dans les affaires d'autrui » ${ }^{12}$. En d'autres termes, ce qui semble au cœur de la culture de sécurité de la Finlande, c'est l'image d'un petit pays qui doit faire preuve de pragmatisme pour demeurer souverain malgré son voisinage avec une grande puissance imprévisible (Browning 2002, 48). En revanche, la neutralité est très ancrée dans la culture de sécurité de la Suède : bien que le pays ne s'y réfère plus que comme à une pratique du passé et qu'il n'en respecte pas les termes à la lettre - notamment le devoir d'être en mesure de se défendre sans aide extérieure -, les Suédois sont très attachés à cette tradition, dans laquelle ils voient un gage de leur souveraineté nationale, mais aussi le fondement de la politique étrangère active au service de la paix dont ils sont fiers. De sorte que si la Suède ne se considère plus officiellement comme neutre, sa politique de sécurité après la fin de la Guerre froide n'en est pas moins imprégnée de l'héritage de la neutralité.

10 Le Parti modéré et le Parti libéral ont très tôt défendu le principe de l'adhésion, auquel le Parti de Gauche et les Verts se sont fermement opposés, tandis que la question a divisé les Chrétiens-démocrates, les Centristes et les Sociaux-démocrates. Une petite majorité des Suédois (52,2 \%) s'est prononcée en faveur de l'adhésion de leur pays à l'Union européenne lors du référendum du 13 novembre 1994.

11 En 2005, 67 \% des Suédois se disent favorables au maintien de la neutralité (Stütz 2005, 144).

12 Cité dans Info-Finlande, « La Finlande et l’OTAN : "être ou ne pas être" ", février 2004. 
Toutefois, l'ordre qui s'était établi après l'effondrement de l'Union soviétique et, surtout, l'équilibre stratégique dans la région nordico-baltique sont profondément remis en cause par la guerre russo-géorgienne qui voit lintervention de l'armée russe en Géorgie en août 2008 et, surtout par l'annexion de la Crimée par la Russie en 2015 dans le contexte de la crise ukrainienne. Cela n'est pas sans impact sur les politiques de sécurité de la Finlande et de la Suède.

\section{L'héritage de la neutralité à l'épreuve de la crise ukrainienne}

Se trouvant sur la route directe de la Russie vers l'UE, la Suède et la Finlande ont une conscience aiguë du danger que représente pour elles une montée des tensions entre, d'une part la Russie et, d'autre part l'Occident, qu'incarnent, aux yeux de Moscou, les États-Unis, l'OTAN et l'Union européenne. Toutefois, si la Russie représente pour eux une menace, à l'instar de l'URSS durant la Guerre froide, les deux pays, loin de renouer avec la politique de sécurité qui avait été la leur durant le conflit bipolaire, adoptent une ligne sécuritaire originale, qui témoigne paradoxalement d'une prise de distance avec leur tradition de neutralité et une fidélité à ce qui fonde leur identité de sécurité.

\subsection{Un environnement menaçant}

La guerre russo-géorgienne ${ }^{13}$, puis la crise ukrainienne ${ }^{14}$ sont une source de fortes préoccupations pour la Suède comme pour la Finlande. En effet, non seulement la Russie, qui a augmenté son budget défense, intervient militairement dans ce qu'elle considère comme son " étranger proche ${ }^{15}$, tentant ainsi de reprendre le contrôle de territoires anciennement soviétiques, mais surtout elle multiplie les provocations dans l'espace baltique.

C'est ainsi que depuis l'éclatement de la guerre en Géorgie, des forces aériennes et navales russes patrouillent régulièrement dans l'espace aérien et à proximité des côtes des États riverains de la Baltique. Moscou organise en outre plusieurs exercices militaires qui sont perçus comme autant de provocations par la Finlande et la Suède. Ainsi, avant même l'éclatement de la crise ukrainienne,

13 Démarrée dans la nuit du 7 au 8 août 2008 par une offensive de l'armée géorgienne en Ossétie du Sud, cette guerre-éclair, qui oppose Tbilissi et Moscou au sujet du contrôle de provinces géorgiennes mais pro-russes, se solde par la signature d'un traité de paix les 15 et 16 août de la même année, sans que cela ne mette fin à la présence militaire russe dans les provinces rebelles.

14 Déclenchée par la décision du président ukrainien Viktor Ianoukovitch, en novembre 2013, de renoncer à conclure un accord d'association avec l'Union européenne, la crise ukrainienne, qui oppose des Ukrainiens prooccidentaux à des Ukrainiens pro-russes, est l'occasion pour Moscou de prendre le contrôle de la province de Crimée.

15 Moscou voit dans son " étranger proche ", c'est-à-dire les pays d'ex-URSS, une sphère d'intérêt vitale. Voir l'entrée « Étranger proche et CEI » du glossaire proposé par le site Géoconfluences 
en mars 2013, la Russie simule, à l'aide de bombardiers intercontinentaux et de chasseurs, une attaque militaire contre Stockholm et contre des bases militaires au sud de la Suède, sans qu'aucun avion de chasse suédois ne réagisse, malgré un état d'alerte théorique $24 \mathrm{~h} / 24 \mathrm{~h}$. Plusieurs années après l'annexion de la Crimée, la Russie n’a pas réduit ses manœuvres dans la Baltique : ainsi, entre mai et juillet 2019, plusieurs avions de la flotte russe ont été interceptés dans la région, y compris des appareils pouvant transporter des charges nucléaires. Le déploiement ponctuel, par la Russie, de missiles tactiques de type « Iskander 2 » dans l'enclave de Kaliningrad met en particulier l'île de Gotland à la merci d'une éventuelle frappe et, plus largement, place la Suède en situation de vulnérabilité, car cela rend potentiellement compliquée une aide extérieure en cas d'escalade. De son côté, la Finlande est particulièrement préoccupée par la présence militaire russe dans la péninsule de Kola. À ces provocations d'ordre militaire s'ajoutent des manœuvres d'intimidation (cyberattaques, achats stratégiques, présence d'agents russes, campagnes de désinformation...). Ces activités constituent, aux yeux des Suédois comme des Finlandais, des menaces hybrides qu'il faudrait prendre au sérieux car elles sont susceptibles de déstabiliser leurs pays.

Par ces manœuvres d'intimidation, Moscou met en garde Helsinki et Stockholm contre toute tentation de rapprochement de l'OTAN. Dans ce contexte, Stockholm n'hésite pas à désigner explicitement la Russie comme une menace potentielle. Ainsi, à l'ouverture du débat sur la politique étrangère au Parlement en 2016, la ministre suédoise des Affaires étrangères Margot Wallström qualifie les agissements de la Russie en Ukraine orientale de " plus grand défi à l'ordre sécuritaire européen depuis la fin de la Guerre froide » (Wallström 2016). Dans leur rapport de 2018, les services secrets suédois (SÄPO) précisent la nature de cette menace : Moscou chercherait, à travers une "doctrine de zone grise ", à affaiblir le pays sans provoquer de conflit armé (Swedish Security Service 2018). Ce constat conduit la Suède à modifier sensiblement sa politique de sécurité. De son côté, si Helsinki ne décrit pas officiellement la Russie comme une menace, la montée en puissance des forces militaires russes et leurs manœuvres dans la Baltique n'en sont pas moins, pour la Finlande, une source d'inquiétudes, comme en témoigne son souci renouvelé d'être en mesure de faire face à toute attaque. Se sentant davantage vulnérables depuis la guerre russo-géorgienne, la Finlande et la Suède ont toutes deux fait évoluer leur politique de sécurité. 


\subsection{Des politiques de sécurité renouvelées}

Tandis qu'au début des années 2000, la Finlande et la Suède ne se considéraient pas en danger, à partir de la guerre russo-géorgienne et, surtout le démarrage de la crise ukrainienne, elles perçoivent leur environnement comme menaçant et font toutes deux évoluer leur pratique sécuritaire en conséquence. Cela étant, si les lignes suivies par les deux pays tendent à converger, des différences demeurent.

\subsubsection{Des postures de sécurité ambiguës}

Renouant avec le positionnement qui était le sien durant la Guerre froide, la Suède cherche à concilier la fidélité au principe de non-alliance, qui est au cœur de sa politique de sécurité depuis le début du XIX ${ }^{\mathrm{e}}$ siècle, avec une intégration plus grande dans les instances de coopération internationale. Alors que la référence à la non-participation du pays à une alliance militaire avait disparu du discours officiel en 2009, sous l'égide du ministre des Affaires étrangères Carl Bildt, elle est reprise dès 2016 par son successeur Margot Wallström, qui décrit le « non-alignement militaire » comme utile pour le pays et, plus largement, pour « la stabilité et [...] la sécurité en Europe du Nord » (Wallström 2016). Dans le même temps, le pays est engagé, du fait des dispositions du Traité de Lisbonne ${ }^{16}$, ainsi qu'en vertu des conclusions du rapport de la commission parlementaire de défense adopté par acclamation le 16 juin $2009^{17}$, dans une politique de solidarité avec les États membres de l'Union européenne et les pays nordiques ${ }^{18}$, notamment en cas d'attaque. Stockholm valorise la coopération internationale comme la meilleure manière d'éviter les menaces à la paix et à la sécurité, tout en cherchant à renforcer ses capacités de défense. Cette position intermédiaire qui fait de la Suède un pays tout à la fois non-allié, solidaire et engagé dans la coopération internationale, relève de ce qu'il est convenu d'appeler la « doctrine Hultqvist », du nom du ministre de la Défense social-démocrate Peter Hultqvist, en poste depuis 2014 (Malmqvist 2018).

16 Entré en vigueur le 1er décembre 2009, le traité de Lisbonne introduit une clause de défense mutuelle - qui dispose que les pays de l'Union européenne sont tenus d'aider un État membre si ce dernier est l'objet d'une agression armée sur son territoire -, ainsi qu'une clause de solidarité en cas de catastrophe d'origine naturelle ou humaine ou d'attaque terroriste.

17 Le rapport indique que la Suède apportera son aide à ses voisins et attend de ces derniers qu'ils fassent de même en cas d'attaque (Rapport du Comité parlementaire de défense 2008/2009).

18 Le 10 avril 2015, les ministres de la Défense suédois, finlandais, norvégien et danois et le ministre des Affaires étrangères islandais émettent une déclaration commune dans laquelle, considérant " l'agression russe contre l'Ukraine et l'annexion illégale de la Crimée » comme " le plus gros défi pour la sécurité en Europe », ils se fixent pour objectif « une coopération plus étroite en Europe du Nord et une solidarité avec les pays baltes » en vue de " renforcer la sécurité dans [la] région » (Euractiv 2015). 
De son côté, la Finlande reste fidèle à la ligne qu'elle a adoptée dès la Guerre froide, en maintenant la défense territoriale au cœur de sa stratégie. Dans le même temps, le pays s'ouvre davantage à la coopération internationale, en particulier à la participation à des opérations de gestion de crise, dans lesquelles il voit une contribution directe à sa sécurité ainsi qu'un outil pour améliorer les compétences et l'interopérabilité de ses forces armées. Surtout, tout en se décrivant comme non-alliée, la Finlande n'exclut pas une candidature à l'OTAN (Prime Minister's Office 2016 ; Rinne 2019), une option dont les conséquences éventuelles ont été analysées par quatre experts ${ }^{19}$ à la demande du ministère des Affaires étrangères (Ministry for Foreign Affairs 2016). Cela étant, la coalition au pouvoir depuis le printemps 2019 a une position ambiguë : tandis qu'une note affirme, conformément aux dispositions du traité de Lisbonne, qu'« en tant qu'État membre de l'Union européenne, la Finlande ne pourrait pas rester neutre s'il y avait une atteinte grave à la sécurité dans son environnement immédiat ou en tout autre endroit en Europe ", une autre note précise que le cabinet du premier ministre Antti Rinne " n'autorisera pas l'utilisation du territoire du pays pour effectuer des actes hostiles dirigés contre un autre pays » (Baltic Monitor 2019). En d'autres termes, tout en se disant prête à combattre aux côtés de ses alliés, elle veille à préserver son territoire en cas de conflit.

La Finlande et la Suède ont donc toutes deux une position ambiguë : tout en s'affirmant non-alliées, elles considèrent leur implication dans la coopération internationale, y compris militaire, comme indispensable pour leur sécurité. Toutefois, alors que la Finlande dit officiellement ne pas être fermée à une éventuelle adhésion à l'OTAN, en Suède, cette possibilité n'est pas ouvertement envisagée, bien que les partis du centre-droit y soient favorables.

\subsubsection{Des capacités de défense renforcées}

À la veille de la crise ukrainienne, la Finlande et la Suède ont des politiques de défense très différentes. Alors que la Finlande a veillé, depuis la fin de la Guerre froide, à maintenir une capacité de défense crédible, en consacrant en moyenne 1,5 \% de son PIB aux dépenses militaires, la Suède a fortement réduit ses dépenses militaires, celles-ci ne représentant que 1,1\% du PIB en 2011. Toutefois, les manœuvres d'intimidation dont ils font l'objet dès 2013 conduisent les deux pays à renforcer leurs capacités de défense.

19 Teija Tiilikainen, chercheuse finlandaise, René Nyberg, ancien diplomate finlandais, Mats Bergquist, ancien diplomate suédois, et François Heisbourg, chercheur français. 
Conformément à sa doctrine, la Finlande n’a jamais cessé de concentrer ses efforts sur ses capacités de défense nationale. C'est ainsi qu'elle a maintenu le service militaire obligatoire (environ 20000 conscrits chaque année) et entretient un vaste réseau de réservistes (près d'un million), se trouvant de la sorte en mesure de mobiliser environ 230000 soldats en temps de guerre, soit plus de $4 \%$ de la population. En 2015, elle décide d'augmenter ses dépenses en matière de défense, avec un objectif de $2 \%$ du PIB en 2022, de manière à pouvoir renforcer ses forces aériennes, favoriser la remise à niveau des militaires... Dans le même temps, elle veille à disposer d'un petit nombre de soldats prêts à participer à des opérations multinationales, sans faire néanmoins de cet objectif une priorité.

En Suède, la situation est différente. Reconnaissant l'incapacité du pays à se défendre seul en cas d'agression militaire, le gouvernement suédois décide, fin 2014, d'entraîner militairement d'anciens conscrits et fait adopter par le Parlement, en avril 2015, le principe d'une augmentation des dépenses de défense de l'ordre de 2,2 \% par an entre 2016 et 2020. En 2017 est rétabli le service militaire (dans la limite toutefois de quelques milliers de conscrits), tandis que le concept de « défense marginale », au cœur de la stratégie suédoise depuis le milieu de la Guerre froide, est supplanté par celui de « défense totale » qui prévoit la mobilisation aussi bien des forces armées que de la société civile en cas d'attaque. Un an plus tard est d'ailleurs distribuée à toute la population une brochure indiquant les mesures à prendre en cas de crise ou de guerre. En 2018, la Suède met sur pied, pour la première fois depuis la fin de la Seconde Guerre mondiale, un nouveau régiment, en vue de protéger l'île de Gotland, territoire le plus proche de la Russie. Cela étant, si le pays cherche officiellement à renforcer sa capacité de défense territoriale, dans les faits, ses forces armées sont essentiellement préparées à la participation à des missions de gestion de crise et la Suède compte avant tout sur une assistance militaire extérieure en cas de conflit.

Depuis le changement de ligne de la Suède en 2014, les politiques de défense finlandaise et suédoise paraissent converger autour de l'impératif de défense nationale. Toutefois, si la Finlande veille à donner corps à la défense territoriale, la capacité de défense de la Suède est en réalité arrimée aux partenariats avec l'OTAN et ses pays membres.

\subsubsection{Des pays engagés dans la coopération internationale}

Dans le contexte de la crise ukrainienne, la Finlande et la Suède ont toutes deux approfondi leur coopération avec l'Alliance atlantique. Elles ont ainsi chacune conclu avec l'OTAN un accord dit de «Host Nation Support» qui permet à 
l'organisation de faire transiter de l'armement, y compris des avions et des navires militaires, sur leur territoire. Parties prenantes de «l'Initiative pour l'interopérabilité avec les partenaires » lancée lors du sommet de l'OTAN de 2014, elles ont toutes deux intégré le programme "Nouvelles opportunités », qui prévoit une coopération plus ciblée sur leurs besoins, en l'occurrence la sécurité dans la région de la mer Baltique. En outre, une déclaration d’intention conclue en mai 2018 annonce le projet d'une entente de défense trilatérale entre la Finlande, la Suède et l'OTAN en vue d'intensifier le trilogue et d'améliorer l'interopérabilité : sont ainsi prévues des rencontres trilatérales régulières, des sessions communes d'entraînement des forces armées, la conduite d'opérations multinationales, etc.

Les deux pays sont également engagés dans des exercices interarmées en vue d'améliorer l'état de préparation de leurs forces armées à recevoir une assistance militaire extérieure. En septembre 2017, la Suède a ainsi organisé l'exercice " Aurora 17 », dans lequel ses soldats se sont entraînés aux côtés d'unités venues du Danemark, d'Estonie, de Finlande, de France, de Lituanie, de Norvège et des États-Unis. La Finlande a décidé d'accueillir un exercice de ce type en 2021.

Toutefois, Helsinki paraît plus prompte que Stockholm à s'engager dans la coopération multinationale en matière de défense. Ainsi, la Finlande participe depuis 2008 à la Force de réaction de l'OTAN, que la Suède n'a rejointe, elle, qu'en 2013. De même, alors que Stockholm s'y était montrée à l'origine réticente, Helsinki a soutenu dès le début la Coopération structurée permanente de l'UE en matière de défense. En outre, à la différence de sa voisine, la Finlande s'est associée en juin 2018 à plusieurs États membres de l'OTAN dans le cadre du Land Battle Decisive Munitions (LBDM), une initiative destinée à favoriser l'acquisition et l'échange d'équipement militaire terrestre afin de réduire les coûts pour tous les participants tout en permettant de développer les capacités des forces de défense. Enfin, elle a rejoint l'European Intervention Initiative lancée par la France en juin 2018, se distinguant ainsi de la Suède.

Depuis la fin des années 2000, la Finlande et la Suède sont donc toutes deux très engagées dans la coopération multinationale en matière de défense, ce qui n'est pas sans brouiller la frontière entre État membre et État partenaire de l'OTAN. 


\subsection{Par-delà les tensions régionales, des politiques de sécurité inchangées ?}

La Finlande et la Suède, si elles demeurent non alliées, n'en sont pas moins plus proches que jamais de l'Alliance atlantique. Cette évolution incontestable de leur politique de sécurité ne témoigne pas pour autant d'une modification en profondeur de leur culture de sécurité.

\subsubsection{Le pragmatisme au coeur de la politique de sécurité finlandaise}

Depuis l'éclatement de la crise ukrainienne, la Finlande a une posture de sécurité assez claire : elle veille à maintenir une capacité de défense territoriale tout en renforçant son implication dans des instances de coopération en vue de ne pas être isolée face à la Russie et d'améliorer l'état de préparation et l'équipement de ses troupes. Dans cette logique, le gouvernement considère ouverte l'option d'une adhésion à l'OTAN, ce qui tranche nettement avec la position qui était la sienne jusqu'aux années 2000. D'ailleurs, lorsqu'on demande aux Finlandais de citer des facteurs qui, selon eux, améliorent la sécurité de leur pays, le non-alignement n'arrive qu'en $6^{\mathrm{e}}$ position (46\%), loin derrière la participation à la coopération nordique de défense (plébiscitée par $83 \%$ des sondés) et la participation à la défense commune de l'UE (70 \%) (ABDI (MTS) 2020). Dans le même temps, plus de $60 \%$ des Finlandais sont opposés à une adhésion à l'OTAN et plus de $40 \%$ voient dans cette éventualité une source d'insécurité pour leur pays ${ }^{20}$. Ces positions sont-elles le signe d'une évolution de la culture de sécurité de la Finlande?

Le discours du président finlandais à l'attention des ambassadeurs, le 25 août 2015, donne à ce sujet un éclairage intéressant (Sauli 2015). Rappelant que les propos du philosophe J. W. Snellman (1863) selon lesquels : « chaque nation agit dans son propre intérêt [...et] ne devrait faire confiance qu'à ellemême » ont constitué le fondement intellectuel de la politique étrangère du pays pendant des décennies, Sauli Niinistö précise ne pas les interpréter comme un appel à « une position isolationniste telle que la neutralité ». Puis, se référant au « réalisme », il met en avant la permanence du sentiment de vulnérabilité qu'est celui de la Finlande, un sentiment lié à la taille (« un pays d'environ cinq millions d'habitants ») comme à la position géographique du pays (« une frontière orientale plus longue que celles des pays de l'OTAN assemblées »). Si, dans les années 1950, cette représentation avait conduit ce pays à opter pour la neutralité

$2064 \%$ des sondés considèrent que la Finlande ne devrait pas chercher à intégrer l'OTAN contre $20 \%$ qui y sont favorables ; $41 \%$ des sondés considèrent qu'une adhésion à l'OTAN réduirait la sécurité de leur pays, $27 \%$ qu'elle augmenterait leur sécurité et 17 \% que cela n'aurait pas d'impact (ABDI (MTS) 2020). 
tout en signant un traité d'amitié et d'assistance mutuelle avec la Russie, dans le contexte de la crise ukrainienne, elle plaide en faveur de la non-alliance couplée à l'entretien d'une capacité de défense autonome et à l'insertion dans des instances de coopération.

C'est donc le même pragmatisme que celui des débuts de la Finlande indépendante qui guide la politique de sécurité d’Helsinki, un pragmatisme qui découle du sentiment de vulnérabilité qui est au cœur de la culture de sécurité du pays.

\subsubsection{La politique de sécurité suédoise à un carrefour}

À l'occasion de sa déclaration annuelle au Parlement, le 12 février 2020, la ministre suédoise des Affaires étrangères Ann Linde, a affirmé : «la politique de sécurité de la Suède reste solidement en place » (Linde 2020). De fait, la « doctrine Hultqvist », qui allie coopération sécuritaire multinationale, politique de non-alignement en temps de paix et solidarité en cas de conflit (Malmqvist 2018) ne diffère pas tant que cela de la ligne qu'a suivie le pays dès le début de la Guerre froide : comme elle le faisait déjà à la fin des années 1940, la Suède assoit sa sécurité sur des partenariats bi- et multilatéraux, préparant ses troupes à recevoir l'assistance militaire extérieure en cas de conflit.

Pour autant, il ne faudrait pas croire que la politique de sécurité de la Suède n'ait pas changé. En premier lieu, Stockholm assume ouvertement son insertion dans des instances internationales de coopération sécuritaire. De plus, ainsi que l'explique le chercheur Mike Winnerstig, bien que le discours officiel mette l'accent sur la continuité, se référant à la non-alliance comme à une source de stabilité et de sécurité régionale ${ }^{21}$, dans les faits, le pays n’a jamais été aussi peu «nonallié » qu'à l'heure actuelle, comme l'illustre par exemple le fait qu'il ait accueilli un exercice de l'OTAN sur son territoire. À cela s'ajoute une évolution notable de l'opinion publique sur les questions de sécurité : la proportion de Suédois qui voient dans la non-alliance un gage de paix et de sécurité est ainsi passée de $57 \%$ à $36 \%$ entre 2011 et 2018 (MSB 2018), tandis que la perception de l'OTAN est de plus en plus positive ${ }^{22}$; les Suédois sont d'ailleurs plus ouverts à la perspective d'une adhésion à l'Alliance atlantique que dans le passée ${ }^{23}$. Dans ces conditions,

21 "Our non-participation in military alliances serves us well and contributes to stability and security in northern Europe", a ainsi affirmé Ann Linde dans sa déclaration de politique étrangère (Linde 2020).

22 D'après une étude conduite au printemps 2019, 63 \% des Suédois ont une opinion favorable de l'OTAN (Fagan and Poushter 2020).

23 En 2018, 31 \% des sondés considèrent qu'une adhésion à l'OTAN aurait des conséquences positives sur la sécurité des pays, contre $35 \%$ qui pensent le contraire. $42 \%$ sont d'ailleurs en faveur d'une adhésion contre $34 \%$ qui y sont opposés (MSB 2018). 
peut-on, à l'instar de Mike Winnerstig, évoquer un « changement [...] radical » de la politique de sécurité suédoise ?

On observe en fait un décalage entre le discours des dirigeants suédois, qui met l'accent sur la continuité de la ligne sécuritaire suédoise, et la réalité d'une politique très tournée vers l'OTAN et d'une opinion publique de plus en plus ouverte à l'éventualité d'une adhésion à l'Alliance atlantique. Une situation ambiguë qui peut être interprétée comme révélatrice du souci constant de Stockholm de ne pas heurter Moscou, mais aussi de ne pas ouvrir de débat sur la politique de sécurité au sein d'une société encline au consensus et qui, forte de l'héritage de la neutralité, considère que le pays se doit d'être une puissance morale (Simons and Manoilo 2019).

\section{Conclusion}

Petits États du Nord de l'Europe, la Finlande et la Suède se réclament toutes deux, historiquement, d'une tradition de neutralité : un choix qui renvoie, pour la première, à un imaginaire de sécurité façonné par la proximité avec une Russie perçue comme menaçante et, pour la seconde, à un désir de rester en paix tout en se projetant comme un acteur au service de la stabilité en Europe.

Bouleversant en profondeur l'équilibre stratégique qui s'était établi dans la Baltique au sortir de la Guerre froide, la crise ukrainienne a conduit les États de la région à adapter leur politique de sécurité. C'est ainsi que la Finlande et la Suède ont fait des choix sécuritaires convergents, en associant non-alliance et participation active à la coopération internationale en matière de sécurité. Ce faisant, elles ont toutes deux pris leurs distances avec l'objectif de neutralité dans un conflit armé, qui avait été au cœur de leur politique sécuritaire depuis les années 1950. Il serait tentant de voir dans ces changements de politique le signe d'une évolution sensible de la culture de sécurité des deux pays. Pourtant, en resituant les pratiques de sécurité d'Helsinki et Stockholm dans l'histoire longue, on ne peut que constater une certaine constance dans leur imaginaire de sécurité : la Finlande continue à se percevoir comme un petit pays toujours en proie à une menace de sa voisine russe, perception qui avait conduit au choix de la neutralité dans les années 1950 et qui justifie aujourd'hui l'intégration très forte dans la coopération sécuritaire internationale, tandis que la Suède continue à se projeter comme un petit pays en paix qui doit contribuer à la paix dans le monde, ce qui découle directement de la posture de neutralité et de la politique de neutralité active qui avait été conduite durant la Guerre froide. On constate donc que l'image que chacun des deux pays a de lui-même - image au fondement 
de la neutralité dans le cas finlandais, image résultant de la neutralité dans le cas suédois - encadre la politique de sécurité nationale de l'un comme de l'autre, lui conférant une direction originale, qui témoigne d'une capacité à évoluer en même temps que de l'impact de l'histoire et de la tradition.

\section{Références bibliographiques}

The Advisory Board for Defence Information (ABDI) (MTS). January 2020. “Finns' Opinions on Foreign and Security Policy, National Defence and Security".

Archer, Toby. 2003. "Keeping out of it: the hangover of Finnish neutralism and the limits of normative commitments. Why are Finns so disinclined to allow Finnish troops to be used elsewhere?” Yearbook of Finnish Foreign Policy 2003: 57-70.

Baltic Monitor. 05.06.2019. “Finland's new coalition government stance on security: to focus less on Russia and war." Warsaw Institute Foundation.

Browning, Christopher S. 2002. "Coming home or moving home? 'Westernizing' narratives in Finnish foreign policy and the reinterpretation of past identities." Cooperation and Conflict XXXVII (1): 47-72.

Clerc, Louis. 2005. « La Finlande entre OTAN et incertitude russe ». Nordiques $7: 22-47$.

Euractiv.10.04.2015. "Nordic nations agree on defence cooperation against Russia."

Fagan, Moira, and Jacob Poushter. 09.02.2020. "NATO seen favorably across Member States". Pew Research Center (Global Attitudes and Trends).

Ganser, Daniele. 07.04.2005. “The secret side of international relations: An approach to NATO's stay-behind armies in Western Europe." Leeds: $55^{\text {th }}$ annual conference of the Political Studies Association.

Goetschel, Laurent. 1998. "The foreign and security policy interests of small States in today's Europe.” In Laurent Goetschel (dir.). Small States Inside and Outside the European Union. Interests and Policies, 13-31. Boston : Kluwer Academic Publishers.

Goetschel, Laurent.1999. "Neutrality, a really dead concept?” Cooperation and Conflict. XXXIV (2): 115-139.

Håkan, Malmqvist. 2018. "Sweden and NATO - 23 years down the road." NATO Review Magazine. Heikka, Henrikki. 2005a. "Republican realism. Finnish strategic culture in historical perspective". Cooperation and Conflict XL (1): 91-119.

Heikka, Henrikki. 2005b. « La défense de l'hégémonie occidentale : un point de vue finlandais ». Nordiques $7: 49-64$.

Kaldor, Mary. 2018. Global Security Cultures. Cambridge/Medford: Polity.

Katzenstein, Peter J. (dir.). 1996. The Culture of National Security. Norms and Identity in World Politics, 1-32. New York: Columbia University Press.

Linde, Ann. 12.02.2020. "The government's statement of foreign policy 2020”. Stockholm.

Longhurst, Kerry. 2004. Germany and the use of force. Manchester/New York: Manchester University Press.

Miles, Lee. 1995. Sweden and European Integration. Hants: Ashgate. 
Ministry for Foreign Affairs. 29.04. 2016. “The Effects of Finland’s Possible NATO Membership. An Assessment". Helsinki.

Molin, Karl. 2000. "The central issues of Swedish neutrality policy". In Michael Gehler und Rolf Steininger (dir.). Die Neutralen und die europäische Integration. 1945 bis 1995, 261-275. Vienne/Cologne/Weimar: Böhlau.

MSB. 18.12.2018. Opinioner 2018.

Nevakivi, Jukka. 1989. "Independent Finland between East and West". In Max Engman and David Kirby (dir.). Finland People, Nation, State, 128-145. London: Hurst \& Company.

Niinistö, Sauli. 25.08.2015. "Speech by President of the Republic at the Ambassador seminar".

Nünlist, Christian. 2013. " La Suède aux prises avec sa politique de défense ". Politique de sécurité : analyses du CSS 138.

Prime Minister's Office. 09.2016. Government Report on Finnish Foreign and Security Policy.

Rapport du Comité parlementaire de défense. 2008/2009. FöU10. Försvarets inriktning.

Report by the Council of State to the Parliament. 06.06.1995. "Security in a Changing World: Guidelines for Finland's Security Policy".

Report by the Council of State to the Parliament. 17.03.1997. "The European Security Development and Finnish Defence".

Rinne, Antti. 11.06.2019. "Prime Minister in Parliament on the Government Programme". Helsinki.

Sakerhetspolisen.se. 2018. Swedish Security Service 2018 Yearbook.

Simons, Greg, and Andrey Manoilo. 2019. "Sweden's self-perceived global role: Promises and contradiction”. Research in Globalization vol.1.

Stütz, Göran. 2005. Opinion 2005. En opinionsundersökning från Stylrelsen för psykologiskt försvar 2005. Stockholm: Styrelsen för psykologiskt försvar.

Sydow, Björn von. 04.04.2018. " Résilience : la Suède planifie la "défense totale" ". NATO Review.

Szymanski, Piotr. 20.03.2020. “The Northern tandem. The Swedish-Finnish defence cooperation". OSW Commentary.

"The Agreement of Friendship, Cooperation, and Mutual Assistance between The Union of Soviet Socialist Republics and The Republic of Finland".

Thibaut, Françoise.1990. La Finlande. Politique intérieure et neutralité active. Paris : LGDJ.

Wallström, Margot. 24.02.2016. "Statement of Government Policy in the Parliamentary Debate on Foreign Affairs 2016”.

Winnerstig, Mike. 2001. "Sweden and NATO”. In Bo Huldt, Teija Tiilikainen et al. (dir.), Finnish and Swedish Security. Comparing National Policies, 76-91. Stockholm: Swedish National Defence College / The Programme on the Northern Dimension of the CFSP. 